\title{
Semi-Procedural Textures Using Point Process Texture Basis Functions
}

\author{
Pascal Guehl*1, Rémi Allègre ${ }^{2}$, Jean-Michel Dischler ${ }^{2}$, Bedrich Benes ${ }^{3}$, and Eric Galin ${ }^{4}$ \\ ${ }^{1}$ ICube - Laboratoire des sciences de l'ingénieur, de l'informatique et de l'imagerie - université de \\ Strasbourg, CNRS : UMR7357 - France \\ ${ }^{2}$ Laboratoire des sciences de l'ingénieur, de l'informatique et de l'imagerie (ICube) - université de \\ Strasbourg : UMR7357, Centre National de la Recherche Scientifique - 300 bd Sébastien Brant - BP \\ 10413 - F-67412 Illkirch Cedex, France \\ ${ }^{3}$ Purdue University - États-Unis \\ ${ }^{4}$ Laboratoire dÍnfoRmatique en Image et Systèmes dínformation - Université Claude Bernard Lyon 1, \\ Centre National de la Recherche Scientifique, Institut National des Sciences Appliquées de Lyon, \\ Institut National des Sciences Appliquées de Lyon - France
}

\begin{abstract}
Résumé
We introduce a novel semi-procedural approach that avoids drawbacks of procedural textures and leverages advantages of data-driven texture synthesis. We split synthesis in two parts: 1) structure synthesis, based on a procedural parametric model and 2) color details synthesis, being data-driven. The procedural model consists of a generic Point Process Texture Basis Function (PPTBF), which extends sparse convolution noises by defining rich convolution kernels. They consist of a window function multiplied with a correlated statistical mixture of Gabor functions, both designed to encapsulate a large span of common spatial stochastic structures, including cells, cracks, grains, scratches, spots, stains, and waves. Parameters can be prescribed automatically by supplying binary structure exemplars. As for noise-based Gaussian textures, the PPTBF is used as stand-alone function, avoiding classification tasks that occur when handling multiple procedural assets. Because the PPTBF is based on a single set of parameters it allows for continuous transitions between different visual structures and an easy control over its visual characteristics. Color is consistently synthesized from the exemplar using a multiscale parallel texture synthesis by numbers, constrained by the PPTBF. The generated textures are parametric, infinite and avoid repetition. The data-driven part is automatic and guarantees strong visual resemblance with inputs. Applications: this work is related to content creation tools for films and video games, especially procedural texture and material synthesis (e.g. Substance Designer), and inverse procedural modeling (e.g inverse shade tree approach).

This paper has been published in the CGF journal (Computer Grapics Forum) in July 2020 and presented at the EGSR conference (Eurographics Symposium on Rendering) in July 2020 where it got an award: Honorable Mention from the Best Papers committee.
\end{abstract}

\footnotetext{
*Intervenant
} 